\title{
Investigation of Titanium Material Deformation for Positioning Stereotactic Navigation System by von Mises Stress Criteria and Finite Element in Engineering
}

\author{
Ö. KARAÇALI* \\ Istanbul University, Department of Mechanical Engineering, Avcllar, Istanbul, 34320 Turkey
}

\begin{abstract}
Positioning stereotactic navigation system (PSNS) is a mechanical system to make a 3D coordinate metallic frame for guiding minor targets in the brain to do biopsy, injection where the treatment is needed. A PSNS head frame is fixed to skull by specially designed pins to keep the head from touching within the frame until the surgery completed. There are although complications in establishing a dependable frame of position, such as bone area that bear a fixed spatial liaison to soft tissues. The fixation or positioning techniques of head frame have been explained in various publications, but related research of mechanical stress/strain analysis about PSNS and pins has not been reported in literature. As a result, the study was undertaken to explore the PSNS head frame and pins by commercial CAD/CAM and ANSYS software packages. The research aim was to develop a three dimensional finite element model and von-Mises criteria model to calculate the mechanical behavior response for the stress distribution around PSNS and related material involving physical surface contact flexural and compressive loading mechanisms. Static analyses were carried out to find the generated stresses and deformation on each part of the studied model. The outcomes reveal that the finite element engineering applied to PSNS model may be employed to calculate the regional distribution of stress-strain-deformation growth in the skull. The knowledge gained from the study points toward some of the engineering advances that are beneficial to stereotactic frame design and mechanical engineers.
\end{abstract}

DOI: 10.12693/APhysPolA.134.409

PACS/topics: finite element analysis, stereotactic apparatus, titanium material mechanical behavior

\section{Introduction}

A stereotactic head frame is similar to metal ring, crown instrument tightly attached to the skull of patient by pins or screws [1]. A semicircular arc for griping a device is fastened to the head frame for biopsy surgery. For the patient's intracranial occlusion, latest study in the area of intraoperative PSNS includes dissimilar models of the head, each of which permits incorporating human skull anatomy [2] with spatially alterable material assets [3]. These prototypes are constructed on physical inspirations like mass-spring techniques [4] or merged energy approach [5]. But, these kinds of models do not have real physical material parameters like soft tissues, though brain biopsy operations have enormous risks [1]. New methods are based on a through physical explanation of the material performance $[3,4]$.

Currently, stereotactic surgeons have applications as morphological, ablative and modular [5]. Titanium alloy also provides ease of use due to the lightness of the mechanical system. In addition, the system must have sufficient strength and dimensional precision [1]. The aim of the research was to investigate the finite element analysis of head frame and to avoid mechanical creep triggered by the bending of pin contact with the cranium and developing loss of torque. PSNS was presented as

*e-mail: ozdogank@istanbul.edu.tr an apparatus that can be helpful in frame locating. Next section explains the computational model of PSNS prototype showed superior performance in pins deformation and von-Mises stress.

\section{Materials and methods for PSNS-pins}

The development process was comprised of geometric, biomechanical and material planning followed by creation and valuation of concept, physical modeling, finite element analysis and justification. Stereotactic guided conformation has been developed for an integrated system for the neurosurgical simulation method. System components include, on each day of treatment, biopsy localization and X-ray computed tomography and radiographic and so on. It is a stereotactic detection system that can be used for examinations and for the positioning unit that can be used for treatment. The research area related to controlling the mechanical properties of the PSNS was identified and developed by finite element modelling for the simulation positioning and optimization shown in Fig. 1 as follows: (1) To estimate the mechanical parameters of developed model. (2) To verify the finite element simulation data obtained from research by matching node displacement with experimental data. (3) To determine the specifications accuracy on the instrument capable of estimate material properties of PSNS.

In Fig. 1, the research method explained including from a real head frame model to analyze virtual data of PSNS model. After selecting a final design concept by refining researched model was supported to create 


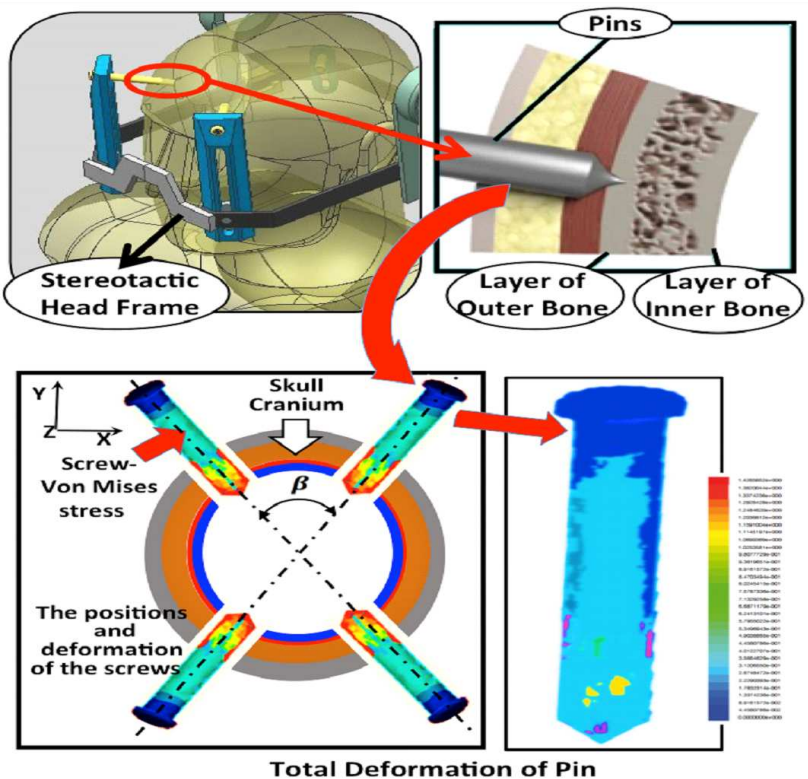

Fig. 1. Pins locations of PSNS deformation.

a quality head frame during design phase. The selected concept was planned geometrically in more detail in the SolidWorks v.18 then assessed by physical model in the ANSYS software version 17 . The geometrical shape corresponding with potential materials was integrated then analyzed. The construction of a model PSNS was mounted on the human skull using the finite element method in engineering. The data related to PSNS are input into the ANSYS. The model consists of 15391,538 elements and 74,781 nodes. The physical properties that were considered were obtained from the scientific literature [5], the load and boundary conditions were applied in agreement with the position of a stereotactic frame fixed to the virtual human head generated in computerized environment. In the analysis of intra-skull stress, two positions were considered to apply four forces of compression to the skull as shown in Fig. 1.

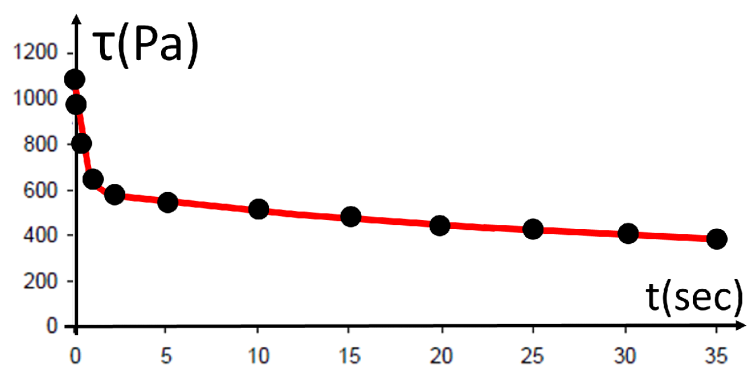

Fig. 2. Shear stress with time.

The proposed material for the frame is titanium. In the frame, the maximum pressure was calculated as $78 \mathrm{MPa}$, at applied screw forces $932 \mathrm{~N}$ with maximum screw strength. The next step of this explorative research was the reverse resolution of the problem, where the material specifications were comprehended from pins. The pins displacement is controlled by force and torque measurements. Figure 2 shows the shear stresses of pins inserted in modeled human skull.

The intra-skull pressure was also considered as shown in Fig. 3. The von-Mises stress and elastic strain patterns in perpendicular axes were calculated for each position, and the distribution of intra-skull stress was obtained. Titanium PSNS apparatus was virtually developed. On the premise for basic laws for material science furthermore constitutive equations for materials were determined to depict those procedures of PSNS application to the skull. The elastic strain was computed in ANSYS-LSDYNA in Eq. (1):

$$
\varepsilon_{e}=\frac{\sqrt{\left(\varepsilon_{1}-\varepsilon_{2}\right)^{2}+\left(\varepsilon_{2}-\varepsilon_{32}\right)^{2}+\left(\varepsilon_{13}-\varepsilon_{21}\right)^{2}}}{\sqrt{2}\left(1+\nu^{\prime}\right)}
$$

where $\varepsilon_{e}$ symbolizes elastic strain in the von-Mises equivalent criteria, $\nu^{\prime}$ stands for the real Poisson ratio, and $\varepsilon_{1}, \varepsilon_{2}$, and $\varepsilon_{3}$ represent the principal strains oriented to the coordinate three axes. The material was viewed as isotropic so the head frame was navigated to extricate the least directional E-modulus [6].

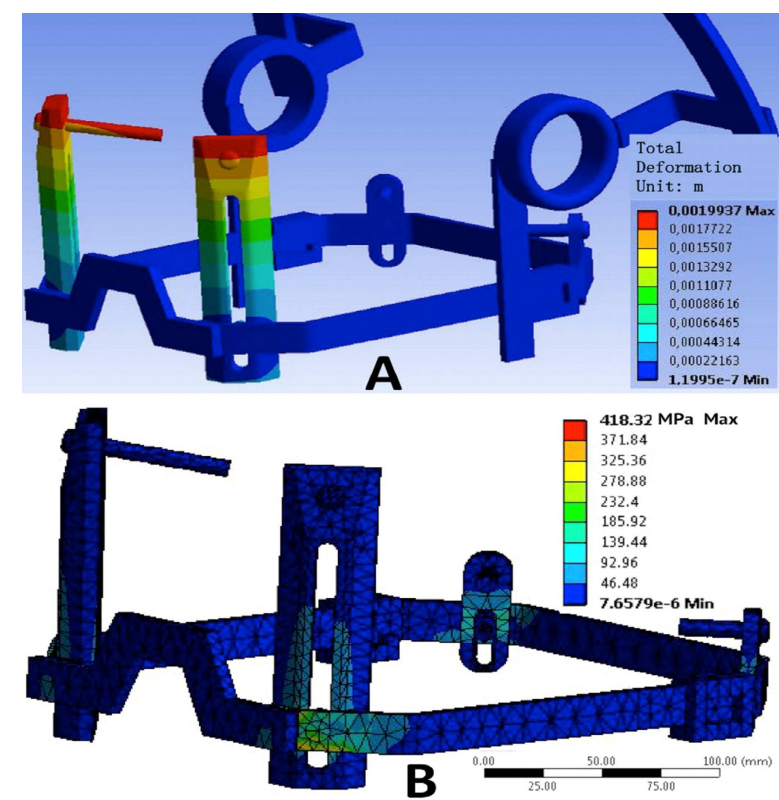

Fig. 3. The total (A) and von-Mises (B) distribution in PSNS.

The screw tips were unlocked without torque. The strength from every screw in the simulation were permitted with move axially through their particular holes, and the energy was set on the holes mantle surface. The thickness of PSNS frame was determined as $8 \mathrm{~mm}$. Those mesh size accepted until 5\% was reached. The deformity was quantified in each diverse screw combinations and position as demonstrated in Fig. 3A. The distortion model in PSNS frame was offered based on the patient head fixation. Required modifications of the stereotactic frame throughout fixation stresses occurred as in Fig. 3B. ASTM F1719-96 and ASTM F1266-89 [7] for Standard 
Performance Specification for Cerebral Stereotactic Instruments standards are used for computational material characteristics in verification of modeling. The total deformation and the equivalent elastic strain is shown in as given Fig. 3a, maximum equivalent von-Mises stresses developed in the PSNS and maximum total deformation in the plates for static conditions were analyzed when inserted in frame under the standing load condition.

Figure 4 shows structural reliability of the PSNS as an important part of the research creating a parametric finite element prototype regarding to finite element method (FEM) outcomes, and resolution for the probability of failure. Stereotactic frame may have greater tolerance of errors, but there are other areas like health where reliability is first unreservedly. Therefore, it is important to test or predict reliability. It is possible to test frame devices, but there is a need to do some functional samples and functional samples are very expensive [5]. This research provides irreplaceable computer simulations and theoretical models for predict reliability.
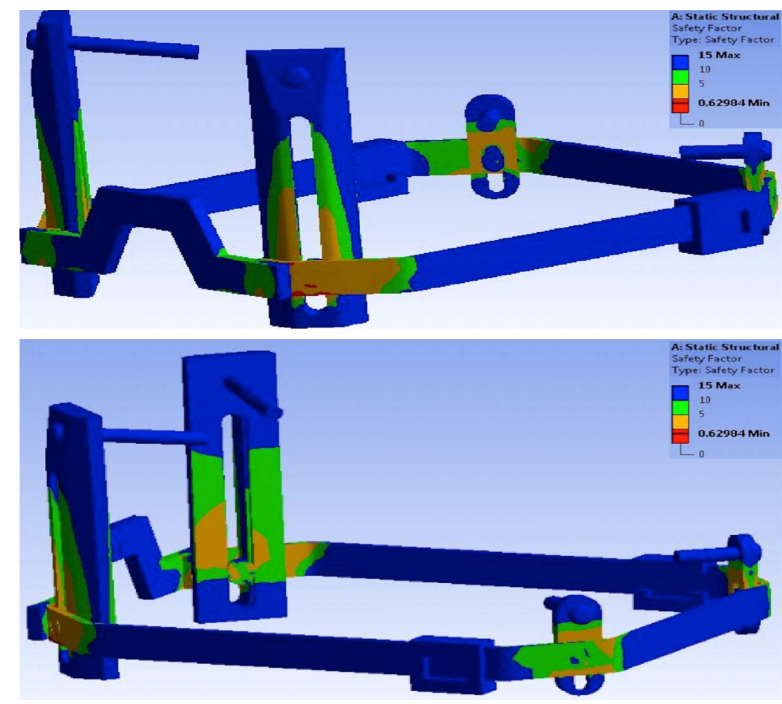

Fig. 4. Reliability factor of the PSNS.

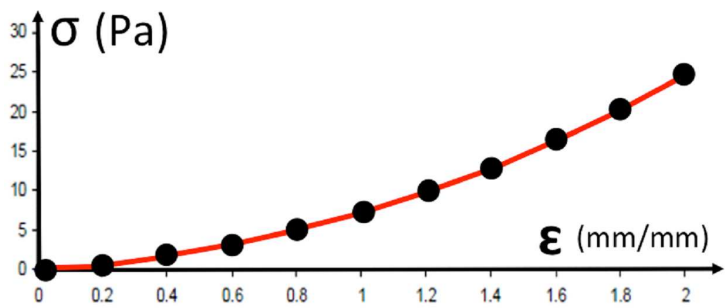

Fig. 5. Static stress of PSNS pin pressure.

Figure 4 displays the basic prediction of reliability using simulation software ANSYS. The reliability model had 1956 elements with 6 degrees of freedom. Stress hardening properties were weighed in the assessment. The PSNS model by FEM limited to 1,9549,0987 nodes and $9,827,994$ elements. Development of reaction surfaces around every outline diminishes errors over deformation estimation, permits an exact assessment about material behavior. The von-Mises equivalent strain under pin pressure was increased around the edge of pin. The static stress of PSNS pin pressure under $1 \mathrm{~mm} / \mathrm{s}$ was calculated in Fig. 5.

\section{Conclusions}

The validation of the simulation and experimental results' comparison was accomplished in the force/displacement profiles in terms of $3 \mathrm{D}$ deformation simulation with the geometry of simulated tissue. The pins of compressive loads of 4,8 , and $12 \mathrm{~N}$ were applied vertically to the skull. The Poisson ratio was set to 0.28 . The Young modulus was fixed to $11.75 \mathrm{GPa}$, density to $1657 \mathrm{~kg} / \mathrm{m}^{3}$. Maximum deformation of frame PSNS was at screw force $500 \mathrm{~N}$, maximum deformation $1.3 \mathrm{~mm}$ with elasticity module $11.72 \mathrm{GPa}$ at $700 \mathrm{~N}$. This study will help to both new and skilled operators of stereotactic radiosurgery, deep brain stimulator implantation, and frame-based biopsy.

The locator attachment of PSNS prototype showed superior performance in pins deformation and von-Mises stress in computational model. These results indicate a longer PSNS lifespan while, the skull received $27 \%$ less von-Mises stress but showed 3\% more total deformation in the locator attachment PSNS model. The analysis of physical loads and displacements allowed identifying the operation steps causing maximal loads on the head restraint PSNS device. The outcomes of the research indicate that the predicative FEM model of PSNS, which incorporate the tips of pins and head tissue to predict the time course and regional distribution of the accompanying regional mechanical characteristics of stress-strain and deformation accumulation of skull.

\section{Acknowledgments}

This work was supported by Scientific Research Projects Coordination Unit of Istanbul University. Project number 27248 .

\section{References}

[1] W.L. Nowinski, Stereotact. Targett. 3, 395 (2009).

[2] P.K. Douglas, Stereotact. Funct. Neurosurg. 83, 1 (2005).

[3] Ö. Karaçall, Acta Phys. Pol. A 131, 584 (2017).

[4] Ö. Karaçalı, Acta Phys. Pol. A 129, 436 (2015).

[5] J.D. Waters, D.D. Gonda, H. Reddy, E.M. Kasper, P.C. Warnke, C.C. Chen, Surg. Neurol. Int. 4, (2013).

[6] D.D. Wang, D. Lau, J.D. Rolston, D.J. Englot, P.K. Sneed, M.W. McDermott, J. Clin. Neurosci. 21, 1538 (2015).

[7] D. Alexander, J. Med. Eng. 12, 1 (2014). 